\title{
Closing the Brief Case: Wound Infection with Plesiomonas shigelloides following a Freshwater Injury
}

(See page 1180 in this issue [doi:10.1128/JCM.02651-15] for case presentation and discussion.)

\section{(D) Morgan A. Pence}

Departments of Laboratory and Pathology, Cook Children's Medical Center, Fort Worth, Texas, USA

\section{ANSWERS TO SELF-ASSESSMENT QUESTIONS}

1. Which oxidase-positive, indole-positive organism is more commonly associated with freshwater injuries?
(a) Stenotrophomonas maltophilia.
(b) Aeromonas hydrophila.
(c) Vibrio vulnificus.
(d) Edwardsiella tarda.

Answer: b. While all of the organisms listed have been isolated from wounds associated with water injuries, the organism most commonly associated with freshwater injuries is A. hydrophila. V. vulnificus is also oxidase positive and indole positive, but it is primarily associated with brackish water or saltwater injuries. S. maltophilia is oxidase negative and indole negative, and $E$. tarda is oxidase negative and indole positive.

2. If a nonhemolytic, non-lactose-fermenting organism isolated from a stool culture agglutinates with Shigella sonnei antisera, which assay can be used to differentiate quickly between Shigella and P. shigelloides?

(a) Growth on MacConkey agar.

(b) Growth on Hektoen enteric agar.

(c) Oxidase.

(d) Urease.

Answer: c. P. shigelloides is oxidase positive, while Shigella is oxidase negative.

3. P. shigelloides is intrinsically resistant to which class of antibiotics?
(a) Penicillins.
(b) Cephalosporins.

(c) Fluoroquinolones.

(d) Aminoglycosides.

Answer: a. P. shigelloides in intrinsically resistant to penicillins due to beta-lactamase production. It is typically susceptible to the other classes listed but may acquire resistance.

\section{TAKE-HOME POINTS}

- P. shigelloides is beta- or nonhemolytic on blood agar and is positive for oxidase, indole, motility, ornithine and lysine decarboxylases, and arginine dihydrolase. Most isolates do not ferment lactose, but rare isolates may ferment lactose.

- The majority of $P$. shigelloides infections occur in the summer months in tropical and subtropical regions.

- P. shigelloides is most commonly associated with gastrointestinal illness, which requires a high infectious dose. Extraintestinal infections are rare but do occur.

- Gastrointestinal infections typically do not require antimicrobial therapy, but if required, fluoroquinolones or trimethoprim-sulfamethoxazole are typically considered the therapy of choice.

- P. shigelloides is intrinsically resistant to penicillins due to beta-lactamase production.

Citation Pence MA. 2016. Closing the Brief Case: Wound infection with Plesiomonas shigelloides following a freshwater injury. J Clin Microbiol 54:1408. doi:10.1128/JCM.02652-15.

Editor: C.-A. D. Burnham

Address correspondence to morgan.pence@cookchildrens.org.

Copyright @ 2016, American Society for Microbiology. All Rights Reserved. 